\title{
O Conceito de Propriedade Termodinâmica
}

\author{
Adalberto B. M. S. Bassi * \\ bassi@iqm.unicamp.br \\ Universidade Estadual de Campinas, Instituto de Química
}

\section{Palavras-Chaves}

Propriedades termodinâmicas

Funções de estado e de processo Equações diferenciais exatas e inexatas Integrais de linha Propriedades extensivas e intensivas Estado termodinâmico

Funções temporais e constitutivas Termodinâmica dos meios homogêneos Termodinâmica dos meios contínuos

\section{Resumo}

São inicialmente mostradas as idéias de grandeza e corpo clássicos, meio contínuo, funções temporal e constitutiva, densidade e taxa, sendo esta última relacionada a noções rudimentares sobre derivadas e funcionais. Em seguida, o conceito de propriedade é indicado, sem a utilização de expressões matemáticas, bem como a classificação das propriedades em intensivas, extensivas e extensivas aditivas. Complementarmente, os conceitos matemáticos de equação diferencial exata e inexata são discutidos e relacionados ao de propriedade. Nesta discussão, aparece o ilogismo e a inoportunidade inerentes ao uso de mais do que um único símbolo matemático para representar o diferencial de uma variável, uso este proveniente de um errôneo conceito de diferencial. $\mathrm{O}$ conceito exato é, então, brevemente referido.

\section{Objetivo}

O conceito de propriedade adquirirá maior clareza e profundidade quando for discutido dentro do contexto da temporal termodinâmica dos meios contínuos, para a qual a aqui considerada termodinâmica dos meios homogêneos é apenas uma natural introdução. Pretende-se apresentar o embasamento deste conceito, dentro do mencionado contexto, porque a compreensão lógica da termodinâmica se tornará muito mais fácil se o seu estudo for precedido pelo completo entendimento da distinção existente entre as propriedades e as demais grandezas termodinâmicas.

Pretende-se, ainda, que na colocação deste embasamento o formalismo matemático e a sua interpretação física se mostrem nitidamente interligados. Para atingir estes objetivos, faz-se necessário expurgar, do modo tradicional de se apresentar noções básicas da termodinâmica, antigos e arraigados vícios de interpretação de conceitos fundamentais do cálculo matemático. Por isto, este trabalho pretende, também, colaborar no estímulo à revisão, na termodinâmica, de tais interpretações incorretas.

\section{Introd ução}

Grandezas clássicas são grandezas cuja existência é suposta por teorias físicas clássicas, tais como mecânica, termodinâmica, eletromagnetismo, elasticidade, acústica, etc. Esta denominação exclui grandezas cujos valores não sejam escalares reais. Exclui, também, grandezas referentes a partículas indivisíveis com dimensões finitas, 
tomadas individualmente, porque os modelos associados a tais partículas, hoje indicados como corretos, não são estudados pelas teorias clássicas ${ }^{1}$, seja ou não contável $^{2}$ o conjunto de valores que a grandeza considerada assumir, nestas partículas.

Por exemplo, não são grandezas clássicas tanto a velocidade angular molecular, como a energia cinética translacional da molécula embora, de acordo com a mecânica quântica, numa molécula a velocidade angular assuma valores discretos, enquanto que a energia cinética translacional, valores contínuos. Mas são grandezas clássicas tanto as velocidades angulares das rodas de um carro, quanto a energia cinética translacional dele. No caso da teoria considerada ser a termodinâmica, a expressão grandeza clássica será substituída por grandeza termodinâmica, para indicar restrição a esta específica teoria.

Corpos clássicos são os objetos naturais estudados pelas teorias clássicas. Eles necessariamente apresentam:

1. Três dimensões ortogonais sendo que, a partir de qualquer ponto do corpo, em cada uma destas dimensões podem ser percorridas, dentro do corpo, distâncias bem maiores do que as dimensões moleculares e bem menores do que as galácticas, portanto podem ser percorridas apenas as distâncias intermediárias características das teorias clássicas.

2. Quantidades de partículas suficientes para validar, dentro do limite de precisão desejado, resultados provenientes de cálculos mecânico estatísticos clássicos (por exemplo, provenientes da distribuição de Maxwell-Boltzmann [1,2]).

As partículas que formam o corpo clássico são contáveis, o número delas é finito e há vácuo entre elas. Elas são indivisíveis, porque a divisão delas alteraria as características da matéria presente no corpo. Mas toda teoria clássica refere-se a um meio contínuo. Meio contínuo é aquele em que, qualquer que seja o instante escolhido, naquele momento verifica-se que os valores de todas as grandezas clássicas englobadas pela teoria considerada diferem, espacialmente, sempre de forma contínua.

A exigência do meio ser contínuo provém do fato de todas as teorias clássicas extrapolarem, para dimensões tão pequenas quanto se queira, comportamentos que, na verdade, são exclusivamente referentes aos corpos clássicos, logo comportamentos inadequados a dimensões tão diminutas. Tal extrapolação é exigida pela aplicação, às variações nos valores das grandezas clássicas entre quaisquer dois pontos do corpo, do cálculo diferencial e integral. Portanto, a continuidade do meio deve ser entendida não como uma realidade natural, mas sim como um artifício necessário à construção de uma teoria matemática que seja experimentalmente comprovada nos corpos clássicos por ela estudados.

Para tratar um corpo clássico como um meio contínuo, a termodinâmica o transforma em um sistema. A exigência de que o meio seja contínuo faz com que, num sistema, a indivisibilidade (das partículas que constituem o corpo clássico) não possa ser atribuída a nenhum volume finito. Logo, num sistema o conceito de partícula indivisível só é aplicável ao ponto matemático, que ocupa o sistema de forma contínua.

Portanto, num sistema a partícula indivisível tem massa e volume nulos, embora a massa e o volume do sistema respectivamente coincidam com os do corpo. Como conseqüência, o conceito de constante de Avogadro, referente aos corpos clássicos, perde o seu significado em sistemas [3].

Reciprocamente, o fato de que, num sistema, cada partícula indivisível seja um dos seus pontos geométricos, garante que um sistema é um meio contínuo exceto, no máximo, em fronteiras. Fronteiras onde aconteçam descontinuidades podem ser as que separam o sistema do seu exterior, mas podem também ocorrer no interior do próprio sistema. Por exemplo, um corpo clássico formado por água sólida e líquida é uma justaposição de corpos cujos interiores apresentam distintos comportamentos termodinâmicos. Mas o sólido passa gradualmente a ser líquido e viceversa, numa região chamada interfacial, cuja espessura é pequena, se comparada com as distâncias existentes nos interiores dos corpos sólido e líquido, mas nunca é nula, como ocorreria se houvesse descontinuidade.

Porém ao extrapolar, para dimensões tão pequenas quanto se queira, os comportamentos dos interiores dos corpos sólido e líquido, se a espessura da região interfacial for suficientemente pequena esta região será, no sistema, substituída por uma superfície traçada no

\footnotetext{
${ }^{1}$ Algumas teorias clássicas (por exemplo, a mecânica estatística de Maxwell-Boltzmann) utilizam modelos matemáticos que consideram a existência de partículas separadas entre si por vácuo, geralmente supostas esferas rígidas. Mas, para tais modelos, as partículas são simples aglomerados de matéria, ou seja, o modelo não exige que elas se constituam em entes unitários indivisíveis.

${ }^{2} \mathrm{O}$ conjunto formado pelos números inteiros positivos é contável, embora contenha infinitos elementos porque é delimitado apenas abaixo, enquanto que o conjunto dos reais positivos inferiores à unidade não é contável, embora seja delimitado acima e abaixo, porque é um conjunto contínuo.
} 
meio da sua espessura. De um lado da superfície haverá o comportamento típico do sólido, do outro será encontrado o comportamento característico do líquido e, na superfície, ter-se-á descontinuidade (como no tratamento dado por Gibbs às interfaces [4]).

\section{Função temporal, função constituti-} va e funcional

A função $y_{\Xi}$ é uma regra que faz com que um ou mais conjuntos ordenados de escalares, denominados vetores $\Xi$ $=\left\langle\mathrm{x}_{1}, \mathrm{x}_{2}, \ldots\right\rangle$, pertencentes ao conjunto $\{\Xi\}$, correspondam a no máximo um único escalar y, pertencente ao conjunto $\{y\}$. O escalar y é chamado imagem, através da função $y_{\Xi}$, do argumento $\Xi$ selecionado, entre os diversos elementos de $\{\Xi\}$. Tal regra não pode depender de qual seja o elemento $\Xi$ escolhido. A igualdade $\mathrm{y}=\mathrm{y}(\Xi)$ indica que o específico vetor $\Xi=\left\langle\mathrm{x}_{1}, \mathrm{x}_{2}, \ldots\right\rangle$, por meio da função $\mathrm{y}_{\Xi}$, determina o específico escalar y. Cabe acrescentar que o escalar $\mathrm{x}_{1}$ pertence ao conjunto $\left\{\mathrm{x}_{1}\right\}$, analogamente ocorrendo para $\mathrm{x}_{2}, \mathrm{x}_{3}, \ldots$ e que o vetor $\Xi$ pode reduzir-se a um único escalar $\mathrm{x}$, caso em que $\Xi=\mathrm{x},\{\Xi\}=\{\mathrm{x}\}, \mathrm{y}_{\Xi}=\mathrm{y}_{\mathrm{x}}$ e a igualdade $\mathrm{y}=\mathrm{y}(\Xi)$ é anotada $\mathrm{y}=\mathrm{y}(\mathrm{x})$.

Conforme já informado, os valores assumidos pelas grandezas termodinâmicas são escalares reais. Quando tais escalares reais forem imagens de funções chamadas temporais, cuja definição é o fato delas apresentarem como argumento exclusivamente o escalar real $t$, que indica o valor do tempo, as correspondentes grandezas termodinâmicas serão denominadas instantâneas. Aliás, o tempo é a única grandeza termodinâmica instantânea cujo valor determina os valores de todas as outras, embora, em geral, valores de outras grandezas termodinâmicas instantâneas não determinem . Portanto, o conjunto dos valores de cada grandeza termodinâmica instantânea é temporalmente ordenado pela correspondente função temporal, ordenamento este que seria perdido se o conjunto de valores $\{t\}$ fosse excluído da teoria, como de fato acontece na termodinâmica atemporal.

O conjunto de valores apresentados por cada grandeza termodinâmica instantânea, porém, existe independentemente de ele mostrar, ou não, ordenamento temporal, ou seja, independentemente da teoria incluir, ou não, o conjunto de valores $\{t\}$. As funções temporais estabelecem, também, uma tabela de simultaneidade entre os valores que as grandezas termodinâmicas instantâneas assumem. Esta tabela, portanto, a cada instante informa qual é o conjunto de valores simultâneos de todas as grandezas termodinâmicas instantâneas. Novamente, o ordenamento temporal destes conjuntos desapareceria numa teoria atemporal, mas os conjuntos em si, ou seja, os agrupamentos de valores compatíveis entre si, continuariam a existir. Logo, numa teoria atemporal a tabela de simultaneidade permaneceria, embora sem ordenamento temporal.

O valor de uma grandeza termodinâmica instantânea pode ser a imagem de uma função que tenha como argumento um conjunto de valores de outras grandezas termodinâmicas instantâneas, simultâneos ao primeiro. Por exemplo, o valor da densidade, $\rho=\mathrm{m} / \mathrm{V}$, é imagem de uma função $\rho_{\Xi}$, sendo $\Xi=<\mathrm{m}, V>$, que tem como elementos valores, da massa e do volume, simultâneos ao valor da densidade. Outro exemplo, válido para cada ponto de um sistema formado por gás suficientemente rarefeito, é $\mathrm{T}=\mathrm{pV}_{\mathrm{m}} / \mathrm{R}$. No primeiro exemplo tem-se uma função constitutiva universal, enquanto que no segundo encontra-se uma função constitutiva específica porque, se o sistema não fosse constituído por gás suficientemente rarefeito, esta específica função $T_{\Xi}$, sendo $\Xi=<p, V_{m}>$ (logo, esta específica regra para se obter $\mathrm{T}$, sabendo-se $\mathrm{p}$ $\mathrm{e} \mathrm{V}_{\mathrm{m}}$ ), perderia validade (a regra apresentada no primeiro exemplo nunca perde a validade).

Portanto, uma função constitutiva permite que, dado um conjunto de valores independentes e simultâneos de algumas grandezas termodinâmicas instantâneas, o valor simultâneo de outra grandeza termodinâmica instantânea seja conhecido, sem o conhecimento da correspondente função temporal. Por isto, as funções constitutivas são extremamente úteis, mesmo numa teoria temporal. Numa teoria atemporal, elas são as únicas informações existentes, além das leis básicas nas quais a teoria se fundamentar.

O operador ou funcional $z_{\eta Y}$ é uma regra que faz com que um ou mais conjuntos ordenados de funções $\mathrm{Y}=\left\langle\mathrm{y}_{1 \Xi}, \mathrm{y}_{2 \Xi}, \ldots\right\rangle$, conjuntos estes chamados argumentos e pertencentes ao conjunto $\{\mathrm{Y}\}$, correspondam a no máximo uma única função $z_{\eta}$ do conjunto $\left\{z_{\eta}\right\}$. A função $z_{\eta}$ é denominada imagem, através do funcional $z_{\eta Y}$, do argumento $\mathrm{Y}$ selecionado. Tal regra não pode depender de qual seja o elemento $\mathrm{Y}$ escolhido, dentro do conjunto $\{\mathrm{Y}\}$. A igualdade $z_{\eta}=z_{\eta}(\mathrm{Y})$ indica que o específico conjunto de funções $Y=\left\langle y_{1 \Xi}, y_{2 \Xi}, \ldots>\right.$, por meio do funcional $z_{\eta Y}$, determina a específica função $z_{\eta}$. Evidentemente, o conjunto ordenado $Y$ pode reduzir-se a uma única função $\mathrm{y}_{\Xi}$, ou seja, é possível que $\mathrm{Y}=\mathrm{y}_{\Xi},\{\mathrm{Y}\}=\left\{\mathrm{y}_{\Xi}\right\}, z_{\eta Y}=z_{\eta y}$ e a igualdade $z_{\eta}=z_{\eta}(\mathrm{Y})$ seja anotada $z_{\eta}=z_{\eta}\left(\mathrm{y}_{\Xi}\right)$.

As funções $\mathrm{y}_{1 \Xi}, \mathrm{y}_{2 \Xi}, \ldots$ são tais que seus vetores argumentos 
$\Xi=\left\langle\mathrm{x}_{1}, \mathrm{x}_{2}, \ldots\right\rangle$ pertençam ao mesmo conjunto $\{\Xi\}$, mas suas imagens escalares y possam ser respectivamente contidas por diferentes conjuntos $\left\{y_{i}\right\}$, sendo $i=1,2, \ldots$. Já a função $\mathrm{z}_{\eta}$ apresenta os vetores argumentos $\eta=\left\langle\mathrm{W}_{1}, \mathrm{~W}_{2}, \ldots\right\rangle$, pertencentes ao conjunto $\{\eta\}$ e as imagens escalares $z$, que são contidas pelo conjunto $\{z\}$. Evidentemente, tanto o vetor $\Xi$ como o vetor $\eta$ podem reduzir-se a um único escalar, respectivamente $\mathrm{x}\left[\Xi=\mathrm{x},\{\Xi\}=\{\mathrm{x}\}\right.$ e $\mathrm{y}_{\mathrm{i} \Xi}=\mathrm{y}_{\mathrm{ix}}$, com a igualdade $\mathrm{y}_{\mathrm{i}}=\mathrm{y}_{\mathrm{i}}(\Xi)$ sendo anotada $\left.\mathrm{y}_{\mathrm{i}}=\mathrm{y}_{\mathrm{i}}(\mathrm{x})\right]$ e $\mathrm{w}[\eta=\mathrm{w}$, $\{\eta\}=\{\mathrm{w}\}$ e $\mathrm{z}_{\eta}=\mathrm{z}_{w}$, com a igualdade $\mathrm{z}=\mathrm{z}(\eta)$ sendo anotada $\mathrm{Z}=\mathrm{z}(\mathrm{w})]$. Além disto, estas simplificações podem, ou não, ocorrer conjuntamente àquela citada no fim do parágrafo anterior a este.

Note que, enquanto uma função é uma regra de transformação de um conjunto de escalares (que se pode reduzir a um único escalar) em um só escalar, um funcional é uma regra de transformação de um conjunto de funções (que se pode reduzir a uma única função) em uma só função. A representação mais geral de uma função é a tabela (às vezes contínua) que associa cada possível vetor argumento à correspondente imagem escalar. Por exemplo, os símbolos matemáticos para seno, logaritmo etc., indicam tabelas específicas (antigamente, eram publicados alguns valores destas tabelas, separados por intervalos convenientes, enquanto que os valores intermediários eram calculados por interpolação). Podese, então, afirmar que um funcional é uma regra de transformação de um conjunto ordenado de tabelas (que se pode reduzir a uma única tabela), em uma só tabela.

\section{Densidade, Taxa e Derivada}

A razão $x / y$, entre os valores simultâneos x e y de duas grandezas termodinâmicas instantâneas é, genericamente, chamada densidade de $x$ em relação a $y$, mas em diversos casos esta razão apresenta denominações específicas. Este é o caso, por exemplo, de $\rho=\mathrm{m} / \mathrm{V}$, que é a densidade de massa em relação ao volume, geralmente chamada apenas densidade ou massa específica, enquanto que $\rho^{-1}=\mathrm{V} / \mathrm{M}$ é a densidade de volume em relação à massa, ou volume específico.

A densidade é o valor, simultâneo a x e a y, de uma outra grandeza termodinâmica instantânea, independentemente do fato da teoria considerada ordenar temporalmente, ou não, a tabela de simultaneidade. Portanto, a densidade é a imagem de uma função constitutiva universal cujo argumento é um par ordenado, função esta que consiste na regra de dividir um elemento do vetor argumento pelo outro, definindo-se o numerador e o denominador da fração de acordo com o ordenamento dos escalares no vetor argumento. Por exemplo, a desigualdade de Clausius afirma que, em cada estado de um sistema termicamente homogêneo ${ }^{3}$ que troque calor, mas não massa com seu exterior, a densidade de potência calorífera [5] em relação à temperatura, grafada $\dot{Q} / \mathrm{T}$, é delimitada por uma cota superior mínima [6].

A um determinado valor $\mathrm{x}$ de uma grandeza termodinâmica instantânea podem corresponder, na tabela de simultaneidade, diversos valores $y_{\alpha}, y_{\beta}, \ldots$ de uma outra grandeza termodinâmica instantânea, desde que os pares $\left\langle\mathrm{x}, \mathrm{y}_{\alpha}\right\rangle,\left\langle\mathrm{x}, \mathrm{y}_{\beta}\right\rangle, \ldots$ refiram-se a momentos diferentes. Portanto, a não ser que seja garantida a existência de uma função constitutiva $\mathrm{y}_{\mathrm{x}}$, o que limitaria o número de pares a apenas um, o conhecimento do valor $\mathrm{x}$ não determina $\mathrm{y}$, logo não determina a densidade $\mathrm{y} / \mathrm{x}$ (evidentemente, o conhecimento de $\mathrm{t}$ determina $\mathrm{x}$, y e $\mathrm{y} / \mathrm{x}$ ). Por exemplo, para um estado termicamente homogêneo de um sistema que troque calor, mas não massa com seu exterior, o conhecimento de T não determina o valor $\dot{Q}$ /T, porque não existe a função $\dot{Q}_{\mathrm{T}}$, mas o conhecimento de $V$ determina o valor $\rho=m / V$, porque existe a função constitutiva constante $m_{v}$.

A razão entre duas variações de valores de grandezas termodinâmicas instantâneas, variações estas que ocorram durante o mesmo intervalo de tempo, é denominada taxa. Se $\mathrm{x}_{\beta}=\mathrm{x}\left(\mathrm{t}_{\beta}\right), \mathrm{x}_{\alpha}=\mathrm{x}\left(\mathrm{t}_{\alpha}\right), \mathrm{y}_{\beta}=\mathrm{y}\left(\mathrm{t}_{\beta}\right), \mathrm{y}_{\alpha}=\mathrm{y}\left(\mathrm{t}_{\alpha}\right), \Delta \mathrm{x}=\mathrm{x}_{\beta}$ $-\mathrm{x}_{\alpha}$ e $\Delta \mathrm{y}=\mathrm{y}_{\beta}-\mathrm{y}_{\alpha}$, então $\Delta \mathrm{y} / \Delta \mathrm{x}$ será a taxa de variação da grandeza cujo valor é y com a grandeza cujo valor é $\mathrm{x}$, desde o instante $t_{\alpha}$ até ao momento $t_{\beta}$. Quando a variação no denominador for $\Delta t=t_{\beta}-t_{\alpha}$, se o numerador for $\Delta \mathrm{x}$ costuma-se chamar a esta taxa de velocidade de variação da grandeza cujo valor é $\mathrm{x}$, desde o instante $\mathrm{t}_{\alpha}$ até ao instante $t_{\beta}$. Por exemplo, $\Delta V / \Delta t$ é a velocidade de variação do volume, no citado intervalo temporal. Numa teoria atemporal, assim como acontece com o conceito de densidade, continua existindo o conceito de taxa, porque a tabela de simultaneidade, embora não mais ordenada pelo tempo, também continua existindo.

Note que a obtenção de uma taxa implica na utilização de um funcional $\mathrm{z}_{\eta Y}$ que apresente, como argumento, o par ordenado de funções $Y=\left\langle x_{t}, y_{t}\right\rangle$, ou apenas a função $Y$ $=\mathrm{x}$ e, como imagem, uma função $\mathrm{z}_{\eta}$ tal que, para $\eta=<\mathrm{t}_{\alpha}$, $\mathrm{t}_{\beta}>$, tenha-se respectivamente $(\Delta \mathrm{y} / \Delta \mathrm{t}) /(\Delta \mathrm{x} / \Delta \mathrm{t})=\Delta \mathrm{y} / \Delta \mathrm{x}=$

${ }^{3}$ Em um sistema termicamente homogêneo a temperatura pode alterar-se, mas a cada instante ela é a mesma em todos os pontos do sistema. 
$\mathrm{z}=\mathrm{z}(\eta)$, ou apenas $\Delta \mathrm{x} / \Delta \mathrm{t}=\mathrm{z}=\mathrm{z}(\eta)$. Note, também, que as duas funções que formam o argumento são, ambas, funções temporais, mas que a função imagem $z_{\eta}$ não é uma função temporal, porque o seu argumento não é um escalar, mas sim um par ordenado de valores do tempo. $\mathrm{Na}$ verdade, a imagem $\Delta y / \Delta x$ ou $\Delta x / \Delta t$ da função $z_{\eta}$, imagem esta que por definição é uma taxa, não é o valor de uma grandeza termodinâmica instantânea, porque $\Delta y / \Delta x$ e $\Delta \mathrm{x} / \Delta \mathrm{t}$ não podem ser imagens de funções temporais, já que não podem ser imagens de funções cujo argumento seja exclusivamente $o$ escalar $t$.

Confundir os conceitos de densidade e taxa, fato que ainda ocorre na literatura termodinâmica atual, evidentemente conduz a absurdos ${ }^{4}$. Densidades e taxas são, respectivamente, os exemplos mais simples de uso de funções constitutivas e de funcionais em termodinâmica. O cálculo diferencial e integral relaciona-se com o conceito de taxa e os operadores de derivação e de integração são os funcionais mais conhecidos das ciências exatas.

De fato, sempre que as funções $x_{t}$ e $y_{t}$ forem contínuas e os seus respectivos gráficos não apresentarem ângulos para todo instante t pertencente a $\Delta \mathrm{t}$, ter-se-á $\dot{y}(\mathrm{t}) / \dot{\mathrm{x}}(\mathrm{t})=\lim _{\Delta \mathrm{t} \rightarrow}$ ${ }_{0}(\Delta \mathrm{y} / \Delta \mathrm{x}), \dot{\mathrm{y}}(\mathrm{t})=\lim _{\Delta \mathrm{t} \rightarrow 0}(\Delta \mathrm{y} / \Delta \mathrm{t})$ e $\dot{\mathrm{x}}(\mathrm{t})=\lim _{\Delta \mathrm{t} \rightarrow 0}(\Delta \mathrm{x} / \Delta \mathrm{t})$, onde t é o instante, pertencente a $\Delta$ t, para o qual tendem $\mathrm{t}_{\alpha}$ e $\mathrm{t}_{\beta}$. Logo, sendo satisfeitas as condições colocadas para $x_{t} e_{t}$, o funcional $\lim _{\Delta t \rightarrow 0} z_{\eta Y}$, onde $z_{\eta Y}$ é o específico funcional definido no penúltimo parágrafo anterior a este, terá como argumento o par ordenado de funções $\mathrm{Y}=\left\langle\mathrm{x}_{\mathrm{t}}, \mathrm{y}_{\mathrm{t}}\right\rangle$, ou apenas $Y=x_{t}$ e, como imagem, uma função $z_{\eta}$ tal que, para $\eta=t$, tenha-se respectivamente $\dot{\mathrm{y}}(\mathrm{t}) / \dot{\mathrm{x}}(\mathrm{t})=\mathrm{z}=\mathrm{z}(\mathrm{t})$, ou apenas $\dot{x}(t)=z=z(t)$. No caso do argumento ser $Y=x_{t}$, $O$ mencionado funcional $\lim _{\Delta t \rightarrow 0} Z_{\eta Y}$ é o funcional derivação total, simbolizado D.

Portanto, a função imagem resultante da aplicação do funcional $\mathrm{D}$ à função $\mathrm{x}_{t}$ é uma função temporal, grafada $\dot{\mathrm{x}}_{\mathrm{t}} \equiv \mathrm{Dx}_{\mathrm{t}}$ e a taxa $\dot{\mathrm{x}}=\dot{\mathrm{x}}(\mathrm{t}) \equiv \mathrm{Dx}(\mathrm{t})=\lim _{\Delta \mathrm{t} \rightarrow 0}(\Delta \mathrm{x} / \Delta \mathrm{t})$, chamada velocidade instantânea de alteração de $\mathrm{x}$, no momento $\mathrm{t}$, é o valor de uma grandeza termodinâmica instantânea. Logo, $\dot{y} / \dot{x}=\dot{y}(t) / \dot{x}(t)$ é uma densidade, ou seja, também é o valor de uma grandeza termodinâmica instantânea. De fato, no parágrafo anterior a este foi mostrado que $\dot{y} / \dot{x}$ é a imagem de uma função temporal.

Havendo a função $y_{x}$ (portanto não existindo, na tabela de simultaneidade, conjuntos que contenham o mesmo valor $\mathrm{x}$ mas valores $\mathrm{y}$ diferentes), sendo esta função contínua e, para todo ponto $\mathrm{x}$ pertencente a $\Delta \mathrm{x}$, não apresentando ângulo o gráfico desta função, tanto numa teoria temporal quanto atemporal ter-se-á a taxa $\lim _{\Delta \mathrm{t} \rightarrow 0}(\Delta \mathrm{y} / \Delta \mathrm{x})=\mathrm{Dy}(\mathrm{x})=$ Dy, onde x é o valor, pertencente a $\Delta \mathrm{x}$, para o qual tendem $\mathrm{x}_{\alpha} \mathrm{e}_{\beta}$. Numa teoria temporal, a taxa Dy é uma grandeza termodinâmica instantânea, porque as exigências necessárias para definir Dy, neste parágrafo colocadas, implicam na satisfação das condições apresentadas no penúltimo parágrafo anterior a este, embora a satisfação destas condições não garanta a obediência às exigências agora colocadas.

Portanto, numa teoria temporal a existência da taxa Dy implica na satisfação da igualdade $\dot{y} / \dot{x}=\dot{y}(t) / \dot{x}(t)=D y(x)$ $=D y$, onde $x=x(t)$, embora a existência da densidade $\dot{y} / \dot{x}$ não garanta que haja a taxa $D y$. Já numa teoria atemporal, $\dot{\mathrm{y}} / \dot{\mathrm{x}}$ nunca existe e Dy pode, ou não, existir. Por exemplo, para um estado termicamente homogêneo de um sistema que troque calor, mas não massa com seu exterior, numa teoria temporal define-se a densidade de potência calorífera em relação à potência térmica, $\dot{Q} / \dot{T}$. Mas, geralmente, não existe a função $\mathrm{Q}=\mathrm{Q}(\mathrm{T})$, portanto não existe a taxa $\mathrm{DQ}(\mathrm{T})$, que seria o valor da derivada do calor trocado em relação à temperatura, quando a temperatura homogênea do sistema fosse $\mathrm{T}$, temperatura esta que ocorreria no instante t ao qual se refere $\dot{Q} / \dot{\mathrm{T}}$.

Porém, se for imposto que nenhuma potência calorífuga seja trocada entre o sistema e o seu exterior, durante o intervalo $\Delta \mathrm{t}$ ao qual pertence $\mathrm{t}$, a primeira lei [5] exigirá que, neste instante, seja satisfeita a igualdade $\dot{U}=\dot{Q}$ . Se for adicionalmente imposto que $U=U(\Xi)$, sendo $\Xi=\left\langle\mathrm{T}, \mathrm{V}, \mathrm{n}_{1}, \ldots, \mathrm{n}_{\mathrm{J}}\right\rangle$ e que as quantidades $\mathrm{n}_{1}, \ldots, \mathrm{n}_{\mathrm{J}}$ de todas as $\mathrm{J}$ substâncias presentes no sistema se mantenham constantes durante o intervalo $\Delta t$, neste intervalo existirá a função derivável $\mathrm{U}_{\mathrm{T}}=\mathrm{Q}_{\mathrm{T}}$, portanto existirá a taxa $\mathrm{DQ}(\mathrm{T})=\mathrm{C}_{\mathrm{v}}$, geralmente denominada capacidade térmica a volume constante. Logo, neste caso específico ter-se-á $\dot{Q} / \dot{T}=$ $\operatorname{DQ}(\mathrm{T})$.

Portanto, numa teoria temporal a densidade $\dot{Q} / \dot{T}$ pode ser considerada uma generalização do conceito de capacidade térmica a volume constante, para estados termicamente homogêneos de sistemas que troquem calor, mas não massa com seu exterior, sistemas estes para os quais não sejam colocadas as imposições apresentadas no parágrafo anterior ao presente. Por outro lado, numa teoria atemporal não existe uma grandeza termodinâmica que consista em tal generalização. Mas perceba a radical

\footnotetext{
${ }^{4}$ Por exemplo, $\rho_{\alpha}=m_{\alpha} / V_{\alpha}$ e $\rho_{\beta}=m_{\beta} / V_{\beta}$ são valores distintos da densidade de massa em relação ao volume e $\Delta \rho=\rho_{\beta}-\rho_{\alpha}$ é a variação desta densidade, ocorrida no intervalo $\Delta \mathrm{t}=\mathrm{t}_{\beta}-\mathrm{t}_{\alpha}$. Por outro lado, $\Delta \mathrm{m} / \Delta \mathrm{V}=\left(\mathrm{m}_{\beta}-\mathrm{m}_{\alpha}\right) /\left(\mathrm{V}_{\beta}-\mathrm{V}_{\alpha}\right) \neq \rho_{\beta}-\rho_{\alpha}$ é a taxa de variação da massa com o volume, para o mesmo intervalo temporal $\Delta \mathrm{t}=\mathrm{t}_{\beta}-\mathrm{t}_{\alpha}$. Não exatamente considerar $\Delta \mathrm{m} / \Delta \mathrm{V}$ e $\rho_{\beta}-\rho_{\alpha}$ iguais entre si, mas sim erros análogos a este são encontráveis em literatura muito recente.
} 
diferença entre a densidade $\dot{Q} / \dot{\mathrm{T}}$ de potência calorífera em relação à potência térmica, que num caso limite transforma-se em capacidade térmica a volume constante e a densidade $\dot{Q} / \dot{T}$ de potência calorífera em relação à temperatura, que numa situação limite transforma-se em velocidade instantânea de variação de entropia. Este é apenas um trivial exemplo do cuidado matemático que a termodinâmica exige.

\section{Propriedade termodinâmica}

Quando a fronteira que separa um sistema do seu exterior for impermeável a tudo o que houver na natureza, salvo ao vácuo (entendido como o nada), às suas dimensões e ao tempo, o sistema será dito isolado. Um observador é um ente imaginário cuja presença, dentro do sistema, não interfere no valor que qualquer grandeza termodinâmica apresente, no sistema ou fora dele. Toda grandeza termodinâmica é direta ou indiretamente perceptível por um observador adequado, caso contrário tal grandeza não seria englobada pela teoria. Entretanto, observadores específicos podem perceber uma grandeza termodinâmica e não outra, como é o caso de um observador situado dentro de um sistema isolado.

Chama-se propriedade a uma grandeza termodinâmica instantânea cuja existência seja perceptível por um observador situado dentro de um sistema isolado e cujos valores, a cada instante, exclusivamente o informem sobre o sistema isolado em questão, naquele momento. Tais valores sempre são relativos a algum referencial conceitualmente bem determinado (por exemplo, metro, quilograma, mol), mas que não precisa ser numericamente bem determinado (por exemplo, o mol não é numericamente bem determinado, mas é conceitualmente bem determinado [3]). Uma vez detectada uma propriedade num sistema isolado, impõe-se que ela também exista, como propriedade, em qualquer outro sistema, isolado ou não. Mas, num sistema isolado, uma grandeza termodinâmica que não seja uma propriedade jamais se transforma em propriedade e vice-versa, enquanto que isto pode ocorrer num sistema não isolado.

De acordo com o conceito adotado não são propriedades, por exemplo, grandezas termodinâmicas exclusivamente relacionadas a movimentos de translação ou de rotação do sistema associado a um corpo rígido [5], porque tais movimentos não seriam percebidos por um observador situado dentro de tal sistema, se este fosse isolado (para percebê-los, o observador precisaria notar pontos externos ao sistema). Não são propriedades, também, grandezas termodinâmicas cujos valores meçam transferências, de qualquer tipo (não se medem transferências de vácuo, de suas dimensões ou de tempo), entre o sistema e o seu exterior.

O tempo não é uma propriedade, porque, se fosse, as velocidades instantâneas de alteração de propriedades seriam valores de propriedades, já que são valores de propriedades tanto diferenças como quocientes entre valores de propriedades, referentes a um mesmo momento. Mas a velocidade instantânea de alteração de uma propriedade informa quanto à tendência dos valores anteriores e posteriores da propriedade serem menores, maiores ou iguais ao seu valor, no instante considerado. Esta informação, por sua vez, contradiz a definição de propriedade. Portanto, as velocidades instantâneas de alteração de propriedades não são propriedades, o que exige que o tempo, também, não seja uma propriedade. Entre as propriedades de um sistema encontram-se, por exemplo, sua massa, que no momento $t$ assume o valor $m$ e seu volume, que no mesmo instante apresenta o valor V.

São valores de propriedades somas, diferenças, produtos ou quocientes entre valores simultâneos de propriedades. Porém, não serão propriedades as grandezas termodinâmicas instantâneas cujos valores, no momento $\mathrm{t}$, forem somas, diferenças, produtos ou quocientes envolvendo uma propriedade e uma não propriedade. Somas, diferenças, produtos ou quocientes entre valores simultâneos de não propriedades podem, ou não, ser valores de propriedades. Por exemplo, imagine-se que fosse $o$ valor de uma propriedade. Neste caso, $V=(V / t) t$ não seria o valor de uma propriedade. Mas, porque $\mathrm{V} / \mathrm{t}$ não é o valor de uma propriedade, $V=(\mathrm{V} / \mathrm{t}) \mathrm{t}$ pode ser o valor de uma propriedade, como de fato é.

Conforme afirmado, as teorias clássicas extrapolam, para dimensões tão pequenas quanto se queira, comportamentos que, na verdade, são exclusivamente referentes aos corpos clássicos, logo comportamentos inadequados para dimensões tão diminutas. Os valores das propriedades, portanto, referem-se às dimensões características das teorias clássicas, mas são extrapolados para valores referentes a pontos matemáticos. Nesta extrapolação, dois comportamentos são observados, conforme será a seguir explicitado.

São chamadas intensivas as propriedades que, ao terem seus valores extrapolados para um ponto matemático, podem apresentar neste ponto qualquer valor real, ou mesmo 
divergir. A cada propriedade intensiva corresponde, no ponto fixo definido pelas coordenadas $\left\langle\mathrm{n}_{1}, \mathrm{n}_{2}, \mathrm{n}_{3}\right\rangle$, uma específica função temporal $x_{t}$, que é a restrição da função $\mathrm{x}_{\Xi}$, onde $\Xi=<\mathrm{t}, \mathrm{x}_{1}, \mathrm{x}_{2}, \mathrm{x}_{3}>$, para $\mathrm{o}$ caso em que as coordenadas espaciais variáveis $\mathrm{x}_{1}, \mathrm{x}_{2}$ e $\mathrm{x}_{3}$ sejam respectivamente fixadas $e m x_{i}=n_{i}$, sendo $i=1,2$ e 3 . São exemplos de propriedades intensivas pressão, concentração, densidade, temperatura, potencial químico, potencial de corrente nula, tensão superficial e seus respectivos inversos.

São chamadas extensivas as propriedades que, ao terem seus valores extrapolados para um ponto matemático, necessariamente apresentam neste ponto valor zero (extensivas aditivas), ou divergem (extensivas não aditivas). Evidentemente, toda propriedade que necessariamente apresente valor zero nesta extrapolação tem, como inversa, uma propriedade que diverge ao ser extrapolada e viceversa.

O valor de toda propriedade extensiva aditiva é qualquer valor real, quando referente a alguma parte finita do sistema, enquanto que o valor de uma propriedade extensiva não aditiva, quando referente à mesma parte, pode ser qualquer valor real, mas também pode divergir. Portanto, para qualquer parte finita do sistema, a cada propriedade extensiva corresponde uma específica função temporal $\mathrm{x}_{t}$. Entre as propriedades extensivas aditivas tem-se volume, massa, quantidade de substância, energia interna, de Helmholtz e de Gibbs, entropia, grau de avanço de reação, área superficial de interface e carga elétrica, enquanto que entre as propriedades extensivas não aditivas encontram-se os respectivos inversos destas propriedades 5 .

\section{Equações diferenciais exatas e} inexatas

Seja a função $z_{1 x}$ diferenciável para $x_{\#}<x<x^{\#}$ e seja, neste intervalo, contínua a função $D z_{1 x}$, derivada de $z_{1 x}$. Para $\mathrm{x}_{\#}<\mathrm{x}<\mathrm{x}^{\#}$ tem-se, então:

$$
z_{1}=z_{1}(x), D z_{1}=D z_{1}(x) \text { e d } z_{1}=D z_{1}(x) d x
$$

Exista, para $\mathrm{x}_{\#}<\mathrm{x}<\mathrm{x}^{\#}$, também a função contínua $\mathrm{z}_{2 \mathrm{x}}$ e a igualdade dela decorrente, $z_{2}=z_{2}(x)$. Multiplicando-se a eq. (1.3) por $z_{2}$, tem-se:
(2) $\quad z_{2}(x) d z_{1}=z_{2}(x) D z_{1}(x) d x$

Seja, também,

$$
\text { (3) } \quad d y=z_{2}(x) d z_{1} \text { e } y_{1}=y_{1}(x)=z_{2}(x) D z_{1}(x)
$$

Substituindo as eqs. (3.1) e (3.2) em (2), pode-se escrever:

$$
\text { (4) } d y=y_{1}(x) d x
$$

Note que, para $\mathrm{x}$ fixo e pertencente ao intervalo mencionado:

1. Porque a função $z_{1 x}$ é diferenciável no intervalo $x_{\#}<x$ $<\mathrm{x}^{\#}$, ambos $\mathrm{dz} z_{1}$ e $\mathrm{dx}$ são diferenciais bem definidos pela eq. (1.3), neste intervalo.

2. Porque, no intervalo $x_{\#}<x<x^{\#}$, as funções $z_{2 x}$ e $\mathrm{Dz}_{1 \mathrm{x}}$ são ambas contínuas, neste intervalo a função também é contínua.

3. Ambos dy e dx são diferenciais bem definidos pela eq. (4), porque esta equação diferencial é uma bem definida reta infinita que passa pela origem do sistema de coordenadas, o que é condição suficiente para definir estes dois diferenciais no intervalo $\mathrm{x}_{\#}<$ $\mathrm{x}<\mathrm{x}^{\#}$. Sublinhe-se, portanto, que não é necessário, para definir estes dois diferenciais, que o coeficiente angular da reta que passa pela origem seja $\mathrm{Dy}=\mathrm{Dy}(\mathrm{x})$. Pela mesma razão, dy e $\mathrm{dz}_{1}$ são diferenciais bem definidos pela eq. (3.1), no intervalo $x_{\#}<x<x^{\#}$.

4. Considere inicialmente a eq. (4). Sejam então lançados num eixo horizontal os reais $\mathrm{x}$, para $\mathrm{x}_{\#}<\mathrm{x}<\mathrm{x}^{\#} \mathrm{e}$, ao $\mathrm{x}$ fixo citado no caput deste parágrafo, corresponda um y arbitrário, medido num eixo vertical. Suponha-se, ainda, que um segmento de curva com comprimento infinitesimal coincida com este ponto $\langle\mathrm{x}, \mathrm{y}\rangle$, sendo a inclinação do segmento dada por $\mathrm{y}_{1}(\mathrm{x})$. Permita-se, agora, que $\mathrm{x}$ se altere continuamente, dentro do seu intervalo de variação. Porque a função $y_{1 x}$ é contínua neste intervalo e porque a altura de todo ponto à direita e à esquerda daquele inicialmente marcado pode ser ajustada em relação à altura deste último, existe uma curva $\mathrm{y}_{\mathrm{x}}$ tal que $\mathrm{y}_{1}(\mathrm{x})=\mathrm{Dy}(\mathrm{x})$, para $\mathrm{x}_{\#}<\mathrm{x}<\mathrm{x}^{\#}$. Portanto, neste intervalo existe uma função diferenciável $\mathrm{y}_{\mathrm{x}}$, tal que $\mathrm{y}_{1}(\mathrm{x})=\mathrm{Dy}(\mathrm{x})$.

5. Se a função $z_{1 x}$ puder ser invertida, de acordo com a eq. (3.1) ter-se-á $d y=z_{2}(x) d z_{1}=z_{2}\left(x\left(z_{1}\right)\right) d z_{1}$ portanto, considerando o colocado no item 4 , existirá a função diferenciável $\mathrm{y}_{\mathrm{z} 1}$ no intervalo $\mathrm{z}_{1 \#}<\mathrm{z}_{1}<\mathrm{z}_{1}^{\#}$, correspondente $\mathrm{a}_{\#}<\mathrm{x}<\mathrm{x}^{\#}$. Porém, se $\mathrm{z}_{1 \mathrm{x}}$ não puder ser invertida, geralmente tal função não existirá.

\footnotetext{
${ }^{5}$ Muitos autores parecem considerar extensivas apenas as propriedades extensivas aditivas, não se manifestando a respeito das extensivas não aditivas.
} 
Este exemplo, embora muito simples, já permite que um importante conceito seja apresentado. De fato, mostrouse que toda equação diferencial com a forma $d y=y_{1}(x) d x$, onde a função $\mathrm{y}_{1 \mathrm{x}}$ é contínua no intervalo $\mathrm{x}_{\#}<\mathrm{x}<\mathrm{x}^{\#}$ que contém o real $\mathrm{x}$, real este que define o valor $\mathrm{y}_{1}=\mathrm{y}_{1}(\mathrm{x})$, é uma equação diferencial exata, porque existe a função $y_{x}$ que, por diferenciação, corresponde a esta equação diferencial. Por outro lado, o adjetivo inexato é reservado para casos em que não exista uma função que, por diferenciação, corresponda à equação diferencial considerada.

Portanto, a equação diferencial $d y=z_{2}(x) d z_{1}$ pode ser inexata, mesmo sendo contínua a função $z_{2 x}$. Mas, se a função $z_{1 x}$ não puder ser invertida e esta equação diferencial for mesmo inexata, desde que $z_{1 x}$ seja diferenciável (conforme suposto no exemplo dado) ela poderá ser transformada numa equação diferencial exata, a saber, poderá ser transformada na eq. (4) por meio da substituição de $\mathrm{d} z_{1}$ pelo seu valor, dado pela eq. (1.3).

Utilizem-se, agora, as funções $z_{1 \Xi}$ e $z_{2 \Xi}$, respectivamente em substituição a $z_{1 x}$ e $z_{2 x}$. Seja, então, $z_{1 \Xi}$ diferenciável para $\Xi_{\#}<\Xi<\Xi^{\#}$, logo seja, neste intervalo,

(5) $\mathrm{d}_{1}=\partial_{\mathrm{x} 1} \mathrm{z}_{1}(\Xi) \mathrm{dx} \mathrm{x}_{1}+\partial_{\mathrm{x} 2} \mathrm{z}_{1}(\Xi) \mathrm{dx}_{2}+\ldots$, onde $\Xi=<\mathrm{x}_{1}, \mathrm{x}_{2}, \ldots>$

Para $\Xi_{\#}<\Xi<\Xi^{\#}$ tenha-se, também, a função contínua $z_{2 \Xi}$. Multiplicando-se a eq. (5) por $z_{2}$ tem-se $z_{2}(\Xi) \mathrm{d} z_{1}$ $=\mathrm{z}_{2}(\Xi) \partial_{\mathrm{x} 1} \mathrm{z}_{1}(\Xi) \mathrm{dx} \mathrm{x}_{1}+\mathrm{z}_{2}(\Xi) \partial_{\mathrm{x} 2} \mathrm{z}_{1}(\Xi) \mathrm{dx}_{2}+\ldots$ e, definindo $\mathrm{dy}=\mathrm{z}_{2}(\Xi) \mathrm{dz} \mathrm{z}_{1}, \mathrm{y}_{1}=\mathrm{y}_{1}(\Xi)=\mathrm{z}_{2}(\Xi) \partial_{\mathrm{x} 1} \mathrm{z}_{1}(\Xi), \mathrm{y}_{2}=\mathrm{y}_{2}(\Xi)=\mathrm{z}_{2}(\Xi) \partial_{\mathrm{x} 2} \mathrm{z}_{1}($ $\Xi), \ldots$, pode-se escrever

(6) $\mathrm{dy}=\mathrm{y}_{1}(\Xi) \mathrm{dx}_{1}+\mathrm{y}_{2}(\Xi) \mathrm{dx}_{2}+\ldots$, onde $\Xi=<\mathrm{x}_{1}, \mathrm{x}_{2}, \ldots>$

A eq. (6) será denominada exata quando

$$
\text { (7) } \mathrm{y}_{1}(\Xi)=\partial_{\mathrm{x} 1} \mathrm{y}(\Xi), \mathrm{y}_{2}(\Xi)=\partial_{\mathrm{x} 2} \mathrm{y}(\Xi), \ldots
$$

Será inexata quando pelo menos uma destas igualdades não for obedecida.

Imponha-se que, numa equação diferencial arbitrária com a forma da eq. (6), $y_{1 \Xi}, y_{2 \Xi}, \ldots$ sejam diferenciáveis. Neste caso, a eq. (6) será exata se e somente se forem satisfeitas todas as igualdades:

$$
\text { (8) } \begin{gathered}
\partial_{\mathrm{x} 1} \mathrm{y}_{2}(\Xi)=\partial_{\mathrm{x} 2} \mathrm{y}_{1}(\Xi), \partial_{\mathrm{x} 1} \mathrm{y}_{3}(\Xi)=\partial_{\mathrm{x} 3} \mathrm{y}_{1}(\Xi), \partial_{\mathrm{x} 2} \mathrm{y}_{3}(\Xi)= \\
\partial_{\mathrm{x} 3} \mathrm{y}_{2}(\Xi), \ldots
\end{gathered}
$$

De fato, um bem conhecido teorema do cálculo diferencial, que informa a invariabilidade das derivadas cruzadas de segunda ordem, quando a ordem de derivação for invertida, mostra que a satisfação das eqs. (7) é suficiente para que as eqs. (8) também sejam satisfeitas. Entretanto, a satisfação das eqs. (8) também é suficiente para que as eqs. (7) sejam satisfeitas. Por outro lado, se $y_{1 \Xi}, y_{2 \Xi}, \ldots$ forem diferenciáveis e não for satisfeita pelo menos uma das eqs. (8), a eq. (6) será inexata e a função $y_{X}$ não existirá ${ }^{6}$.

Diferenciais são reais arbitrários que satisfazem a equação definidora, para $x$ ou $\Xi$ fixos [eqs. (4) ou (6) respectivamente], independentemente de ser exata ou inexata tal equação definidora $[6,7]$. Porém, compêndios tradicionais de termodinâmica, além de confundirem diferenciais com infinitésimos [6,7], também costumam distinguir os diferenciais entre si, de acordo com o fato deles serem definidos por meio de equações diferenciais exatas ou inexatas. Sublinhe-se que é a equação que é exata ou inexata, não o diferencial. Por isto, não tem sentido utilizar símbolos diferentes para o diferencial (embora a própria IUPAC se refira a tais símbolos [8]), de acordo com o fato de a equação definidora ser exata ou inexata, conforme pode ser confirmado lendo-se qualquer livro de equações diferenciais (veja-se, por exemplo, um dos mais renomados [9]).

Isto pode, também, ser percebido por meio de uma simples interpretação geométrica do que seja exatidão e inexatidão. Para isto, considere o espaço tridimensional definido pelos eixos ortogonais referentes a $x_{1}, x_{2}$ e y, e seja $\Xi=<x_{1}, x_{2}>$. Para $x_{1}$ e $x_{2}$ fixos, dy $=y_{1}(\Xi) d x_{1}$ $+\mathrm{y}_{2}(\Xi) \mathrm{dx}_{2}$ é a equação de um plano infinito que pertence a este espaço e passa pela sua origem (suponha que $\mathrm{dx}_{1}$ e $\mathrm{dx}_{2}$ sejam variáveis independentes, respectivamente colineares aos eixos referentes a $x_{1}$ e $x_{2}$, e que dy seja a variável dependente, colinear ao eixo referente a y). Imagine que uma superfície, com área infinitesimal, tangencie um plano paralelo àquele definido pela equação diferencial fornecida, num ponto com coordenada y arbitrária mas cujas outras duas coordenadas sejam respectivamente os reais fixos $\mathrm{x}_{1}$ e $\mathrm{x}_{2}$, que determinam os coeficientes constantes do plano, $\mathrm{y}_{1}(\Xi)$ e $\mathrm{y}_{2}(\Xi)$.

Suponha, agora, que $\mathrm{x}_{1}$ e $\mathrm{x}_{2}$ variem de modo contínuo, dentro de intervalos definidos. Somente se nos citados intervalos a igualdade $\partial_{\mathrm{x} 1} \mathrm{y}_{2}(\Xi)=\partial_{\mathrm{x} 2} \mathrm{y}_{1}(\Xi)$ for obedecida, existirá então uma superfície $y_{\Xi}$ com área finita e tal que o plano tangente à mesma, no ponto $\left\langle\mathrm{x}_{1}, \mathrm{x}_{2}, \mathrm{y}\right\rangle$, seja

\footnotetext{
${ }^{6}$ Este é o chamado "teste de exatidão", apresentado na maioria dos livros de termodinâmica, freqüentemente de modo tal que parece ser a própria definição de equação diferencial exata, ou melhor, de "diferencial exato", uma vez que muitos livros classificam não a equação diferencial, mas sim o diferencial.
} 
paralelo ao plano dy $=\mathrm{y}_{1}(\Xi) \mathrm{dx}_{1}+\mathrm{y}_{2}(\Xi) \mathrm{dx} \mathrm{x}_{2}$, para todo $\mathrm{x}_{1}$ e $\mathrm{x}_{2}$ pertencente ao seu respectivo intervalo de variação. Para visualizar o motivo geométrico desta afirmação, deve-se lembrar que $\partial_{\mathrm{x} 1} \mathrm{y}_{2}(\Xi)=\partial_{\mathrm{x} 2} \mathrm{y}_{1}(\Xi)$ se e somente se $\mathrm{y}_{2}(\Xi)=\partial_{\mathrm{x} 2} \mathrm{y}(\Xi)$ e $\mathrm{y}_{1}(\Xi)=\partial_{\mathrm{x} 1} \mathrm{y}(\Xi)$, onde $\mathrm{y}_{\Xi}$ é a superfície considerada.

Mas $\partial_{\mathrm{x} 1} \partial_{\mathrm{x} 2} \mathrm{y}(\Xi)$ é a taxa de variação, para alteração na direção e sentido de máximo acréscimo em $\mathrm{x}_{1}$, da inclinação da tangente (no ponto $<\mathrm{x}_{1}, \mathrm{x}_{2}, \mathrm{y}>$ da superfície $\mathrm{y}_{\Xi}$ ) que apresenta a direção e o sentido de máximo acréscimo em $\mathrm{x}_{2}$, sendo análoga a interpretação de $\partial_{\mathrm{x} 2} \partial_{\mathrm{x} 1} \mathrm{y}(\boldsymbol{\Xi})$. Para a existência da superfície $\mathrm{y}_{\Xi}$, é geometricamente necessário que estas duas taxas se igualem. Aliás, esta é a explicação geométrica para o já mencionado teorema que afirma ser $\partial_{x 1} \partial_{x 2} y(\Xi)=\partial_{x 2} \partial_{x 1} y(\Xi)$. Esta condição de existência da superfície $y_{\Xi}$ é facilmente visualizável num espaço tridimensional, mas por razões geométricas não apresenta análogo no espaço bidimensional. De fato, para este último já se afirmou que toda equação diferencial com a forma $\mathrm{dy}=\mathrm{y}_{1}(\mathrm{x}) \mathrm{dx}$ será uma equação diferencial exata, se $\mathrm{y}_{1}=\mathrm{y}_{1}(\mathrm{x})$ for contínua.

Note-se que a família de planos dy $=\mathrm{y}_{1}(\Xi) \mathrm{dx}_{1}+\mathrm{y}_{2}(\Xi) \mathrm{dx} \mathrm{x}_{2}$, que corresponde ao conjunto de pares ordenados $\Xi=<x_{1}, x_{2}>$ para todos os valores permitidos $x_{1}$ e $x_{2}$, existe independentemente da existência adicional de uma superfície que tangencie um plano paralelo a cada um destes planos, no ponto que apresente como coordenadas independentes o par $\Xi=<\mathrm{x}_{1}, \mathrm{x}_{2}>$, par este que determina a inclinação do plano tangenciado. Ocorrendo esta existência adicional, dy $=\mathrm{y}_{1}(\Xi) \mathrm{dx}_{1}+\mathrm{y}_{2}(\Xi) \mathrm{dx}_{2}$ será uma equação diferencial exata. Não ocorrendo, a equação diferencial será inexata. Este, evidentemente, é o caso mais comum.

Claramente, tal existência adicional é uma característica da equação diferencial, ou seja, dos coeficientes $y_{1}(\Xi)$ e $\mathrm{y}_{2}(\Xi)$ das variáveis independentes, não sendo uma característica das próprias variáveis $\mathrm{dx}_{1}, \mathrm{dx}_{2}$ e dy. Percebese que a interpretação de diferenciais, como sendo variáveis que definem entes geométricos (retas, planos etc.) [10], dificilmente levaria a se distinguir diferenciais entre si, chegando-se ao extremo de símbolos diferentes serem utilizados. Isto foi conjuntamente causado pela confusão entre diferenciais e infinitésimos, pelo esquecimento da necessidade de satisfação da equação definidora, por um falho conceito de infinitésimo $[6,7]$ e pela total falta da visualização geométrica aqui apenas esboçada.

\section{Conclusão}

O conceito de estado é matematicamente bem definido, sendo o menor conjunto de valores de propriedades do sistema, no instante $t$, suficiente para que os valores de todas as propriedades do sistema, admitidas pela teoria termodinâmica considerada, sejam conhecidos naquele momento. Suponha que o vetor $\Xi$ represente o estado do sistema. Por exemplo, num sistema que não troque massa com seu exterior e que seja homogêneo em relação a todas as suas propriedades intensivas, freqüentemente em química é conveniente considerar $\Xi=<\mathrm{T}, \mathrm{p}, \mathrm{n}_{1}, \ldots, \mathrm{n}_{\mathrm{J}}>$, onde $T$, $p$ são respectivamente a temperatura e a pressão homogêneas do sistema no instante considerado, enquanto que $\mathrm{n}_{1}, \ldots, \mathrm{n}_{\mathrm{J}}$ são as quantidades das $\mathrm{J}$ espécies químicas distintas presentes no sistema, naquele momento.

A definição de estado indica que, se $\mathrm{x}$ for o valor de qualquer propriedade do sistema no instante $t$ e $\Xi$ for o estado do sistema naquele momento, existe a função constitutiva $\mathrm{x}_{\Xi}$, logo $\mathrm{x}=\mathrm{x}(\Xi)$. Por outro lado, se no mesmo instante y for o valor de uma grandeza termodinâmica instantânea que não seja uma propriedade, nada obriga a que exista a função constitutiva $\mathrm{y}_{\Xi}$ e, como seria de se esperar, geralmente ela não existe. Evidentemente, ao se diferenciar $\mathrm{x}_{\Xi}$ obter-se-á uma equação diferencial exata, enquanto que equações diferenciais envolvendo o diferencial dy e diferenciais de propriedades presentes em $\Xi$, ou mesmo de outras propriedades, em geral serão inexatas.

Por causa disto, os autores muitos livros tradicionais de termodinâmica chamam $\mathrm{x}$ de função de estado e $\mathrm{y}$ de função de processo, o que é correto embora este autor prefira a denominação propriedade, porque o seu significado físico, sem a utilização de equações matemáticas, é mais intuitivo do que o de função de estado. Aliás, muitos livros tradicionais preferem o nome propriedade. Quando esta escolha for feita, as funções de processos serão definidas por exclusão, ou seja, serão todas as grandezas termodinâmicas, salvo as propriedades.

A crítica ao símbolo de "diferencial inexato" não se prende exclusivamente a um apego à mais elementar lógica matemática. Acontece que o uso deste símbolo ajuda a inculcar nos estudantes todo o conjunto de erros matemáticos que infesta os livros de termodinâmica [6,7]. $\mathrm{O}$ teste de exatidão mostrado, presente em quase todos os livros de termodinâmica, é realmente muito útil para se distinguir os dois tipos de equações diferenciais, nada mais do que isto sendo necessário. 
Referências

1. Boltzmann, L., Vorlesungen über Gastheorie, I; Barth: Leipzig, 1896.

2. Boltzmann, L., Vorlesungen über Gastheorie, II; Barth: Leipzig, 1898.

3. Bassi, A. B. M. S., Quantidade de Substância, ChemKeys 2005.

4. Gibbs, J. W., The Collected Works of Josiah Willard Gibbs, Longmans, Green: New York, 1928.

5. Bassi,A.B.M.S., As Duas Expressões Fundamentais da Termodinâmica, http://www.chemkeys.com, 2006.

6. Truesdell, C. A., Rational Thermodynamics; Springer-Verlag: New York, 1984.

7. Bassi, A. B. M. S., Matemática e Termodinâmica, ChemKeys, 2005.

8. I. Mills, I.; Cvitas, T.; Homann, K.; Kallay, N.; Kuchitsu, K., Quantities, Units and Symbols in Physical Chemistry (The Green Book); Blackwell Science: Oxford, 1993.

9. Ince, E. L., Ordinary Differential Equations; Longmans, Green: London, 1926.

10. Apostol, T. M., Mathematical Analysis, A Modern Approach to Advanced Calculus; Addison Wesley: Reading, 1957. 